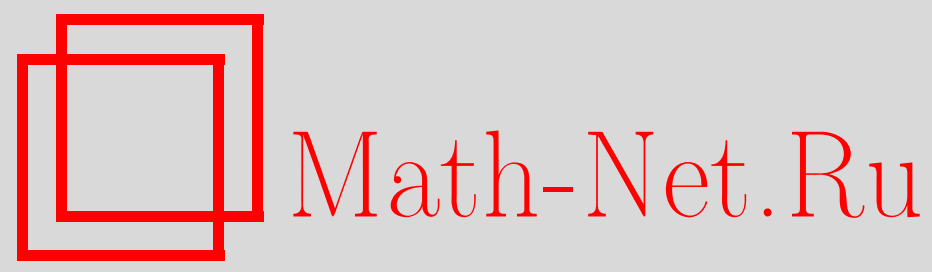

М. Э. Михайлов, Разложение конечных псевдометрических пространств, Матем. заметки, 1998, том 63, выпуск 2, 225234

DOI: https://doi.org/10.4213/mzm1269

Использование Общероссийского математического портала Math-Net.Ru подразумевает, что вы прочитали и согласны с пользовательским соглашением http://www. mathnet.ru/rus/agreement

Параметры загрузки:

IP : 54.198 .64 .247

26 апреля 2023 г., 15:26:07

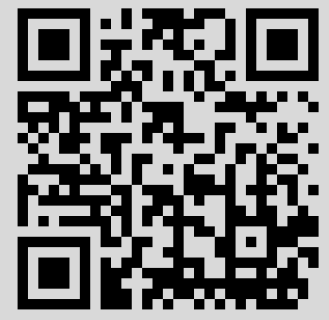




\title{
РАЗЛОЖЕНИЕ КОНЕЧНЫХ ПСЕВДОМЕТРИЧЕСКИХ ПРОСТРАНСТВ
}

\section{М.Э. Михайлов}

\begin{abstract}
Для псевдометрик определено свойство разложимости (неразложимости), которое состоит в возможности (невозможности) представить псевдометрику в виде суммы двух псевдометрик любым иным способом, кроме деления всех расстояний в одинаковой пропорции. Доказано, что при заданном конечном числе точек $n$ существует набор из конечного числа неразложимых псевдометрик (базис), который порождает путем линейного комбинирования с неотрицательными коэффициентами множество всех псевдометрик. Перечислены все компоненты базиса для $n \leqslant 7$. Вьведена процедура определения разложимости или неразложимости любого конечного (состоящего из конечного числа точек) псевдометрического пространства. Установлены некоторые признаки разложимости и неразложимости.

Библиография: 6 названий.
\end{abstract}

Простые аксиомы, определяющие метрическое пространство, допускают бесчисленное разнообразие метрик. Однако это разнообразие можно привести в систему. В данной работе показьвается, как все возможные метрические и псевдометрические пространства, образованные конечным числом точек, порождаются более узким множеством так называемых неразложимых пространств. Понятие метрики при этом пришлось расширить до псевдометрики, поскольку оказалось, что без привлечения псевдометрических пространств эта задача не решается.

1. Псевдометрические и метрические пространства. Псевдометрическое пространство определяют как множество, каждой паре элементов (точек) которого соответствует вещественное число, назьваемое расстоянием и удовлетворяющее следующим аксиомам [1] (расстояние от точки $a$ до точки $b$ обозначаем $r(a, b)$ ):

1) неотрицательность: $r(a, b) \geqslant 0$;

2) симметричность: $r(a, b)=r(b, a)$;

3) рефлексивность: $r(a, a)=0$;

4) неравенство треугольника: $r(a, b) \leqslant r(a, c)+r(b, c)$.

Частньй случай псевдометрических пространств - метрические. Дополнительное требование к ним - расстояние между разными точками не должно равняться 0 [1]. Обычно его включают в аксиому 3 ).

Хотя условия 1), 2) выводятся из 3), 4) (см. [2]), на практике удобно считать их все аксиомами: справедливость аксиомы 4) проверить значительно легче, если перед этим установлена справедливость аксиом 1), 2).

Псевдометрические пространства будем назьвать просто пространствами. Пары точек будем назьвать сторонами, а расстояния между точками - длинами сторон. 
Назовем точки слившимися, если расстояние между ними равно 0. Слитность есть отношение эквивалентности [3]. Симметричность и рефлексивность следуют из аксиом 2), 3), транзитивность следует из неравенства треугольника: если две точки слиты с третьей, то они слиты и между собой [2]. Группу слившихся точек будем называть кратной вершиной. Точку, не входящую в какую-либо кратную вершину, будем называть одиночной верииной.

Из неравенства треугольника следует, что точки, входящие в кратную вершину, находятся на одинаковом расстоянии от любой другой точки. Таким образом, единственньй признак, выделяюший метрические пространства среди псевдометрических, отсутствие кратных вершин [2]. В дальнейшем будем говорить о расстояниях между вершинами, подразумевая расстояния между входящими в них точками, а пару вершин назьвать стороной.

Если для трех точек $a, b, c$ неравенство треугольника превращается в равенство, причем $r(a, b) \neq 0, r(b, c) \neq 0$, то большую сторону ac будем назьвать составной, а меньшие стороны - составляющ,ими. Несоставные стороны с ненулевой длиной будем называть әлементарными.

Число точек в пространстве будем назьвать его рангом. Пространства, состоящие из конечного или бесконечного числа точек, будем называть соответственно конечным. или бесконечными.

\section{2. Графическое изображение конечных псевдометрических пространств.}

Конечные псевдометрические пространства можно представлять табличным способом: на пересечении строк и столбцов, соответствующих различным точкам, записываются расстояния между ними.

Мы же будем, как и в [4], изображать конечные пространства в виде взвешенных графов, т.е. каждому ребру приписывать положительное число - его длину. Здесь и далее имеются в виду простые графы, т.е. неориентированные, без петель и кратных ребер [5]. Вершины графа соответствуют вершинам пространства, ребра - элементарным сторонам. Расстояния проставляются рядом с ребрами. Если расстояния не указаны, подразумевается, что они равны 1 . Одиночную вершину изображаем точкой, кратную кружком, в котором размешено соответствующее число точек. От кратной вершины проводится только один комплект ребер, поскольку слившиеся точки дают идентичные наборы расстояний. Составные стороны в графе не изображаются, но их можно вычислить: в [4] доказано, что длина любой составной стороны равна длине кратчайшего маршрута, соединяюшего ее конщы. Примером служит рис. 1, где одно и то же пространство задано табличным и графическим способом.

\begin{tabular}{|c|c|c|c|c|c|c|c|}
\hline & $\alpha$ & $\beta$ & $\gamma$ & $\delta$ & $\varepsilon$ & $\zeta$ & $\eta$ \\
\hline$\alpha$ & 0 & 0 & 2 & 2 & 1 & 1 & 1 \\
\hline$\beta$ & 0 & 0 & 2 & 2 & 1 & 1 & 1 \\
\hline$\gamma$ & 2 & 2 & 0 & 0 & 1 & 1 & 1 \\
\hline$\delta$ & 2 & 2 & 0 & 0 & 1 & 1 & 1 \\
\hline$\varepsilon$ & 1 & 1 & 1 & 1 & 0 & 2 & 2 \\
\hline$\zeta$ & 1 & 1 & 1 & 1 & 2 & 0 & 2 \\
\hline$\eta$ & 1 & 1 & 1 & 1 & 2 & 2 & 0 \\
\hline
\end{tabular}

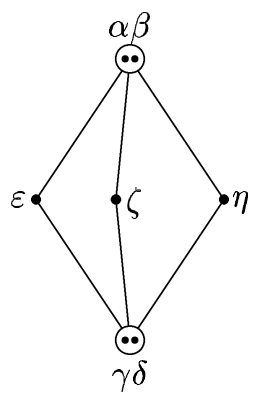

Рис. 1 
Поставим обратную задачу: каким требованиям должен удовлетворять произвольный взвешенньй связньй граф, чтобы служить изображением метрического пространства? Если расстояние между вершинами определить как длину кратчайшего соединяющего их маршрута, то, как показано в [4], аксиомы метрики будут выполняться в любом случае. Кроме того, требуется, чтобы в графе не изображались составные стороны, а длина каждого ребра равнялась расстоянию между его концами. Нетрудно проверить, что для этого необходимо и достаточно следующее условие: каждое ребро должно являться единственным кратчайшим марирутом межсду своими вериинами. В частности, оно вьполняется автоматически, если все ребра равны по длине. Очевидно, это условие необходимо и достаточно и для изображения псевдометрического пространства, если в графе допустить кратные вершины.

При задании метрики на графах часто допускают бесконечные расстояния для вершин, не связанных никаким маршрутом (см., например, [4]). В данной работе бесконечные расстояния не допускаются по формальньм соображениям: $+\infty$ не входит в поле вещественньх чисел.

3. Операции над конечными псевдометрическими пространствами. На множестве пространств одного ранга, между точками которых установлено взаимно однозначное соответствие, можно определить следующие операции.

1. Умножение на коэффишиент. Длины всех сторон пространства $A$ умножаются на одно и то же неотрицательное число $c$. В результате получаются длины соответствующих сторон пространства $c A$. Операция, очевидно, всегда выполнима. Пространства, получаемые друг из друга умножением на коэффициент, будем называть подобными.

2. Сложение. Длина каждой стороны пространства-суммы $C=A+B$ равна сумме длин соответствуюших сторон пространств-слагаемых $A$ и $B$. Операция всегда вьполнима: сумма двух псевдометрик есть псевдометрика [2]. Обратная операция - вычитание - выполняется, очевидно, не всегда.

Представление пространства $A$ в виде линейной комбинации $A=\sum c_{i} B_{i}\left(c_{i}-\right.$ положительные коэффициенты) будем называть разло жением. Любое пространство можно разложить на два подобных ему (и друг другу), разделив все стороны в одинаковой пропорции. Нас, однако, будет интересовать разложение на неподобные пространства. Пространства, которые нельзя разложить на два неподобных, будем называть неразложиммыми .

4. Разложение конечных псевдометрических пространств. В псевдометрическом пространстве для каждой тройки точек $a, b, c$ должны выполняться три неравенства:

$r(a, c)+r(b, c)-r(a, b) \geqslant 0, \quad r(a, b)+r(a, c)-r(b, c) \geqslant 0, \quad r(a, b)+r(b, c)-r(a, c) \geqslant 0$.

Троек точек в пространстве ранга $k$ содержится $k(k-1)(k-2) / 6$, поэтому должны выполняться $k(k-1)(k-2) / 2$ неравенств с $k(k-1) / 2$ неизвестными. Множество всех псевдометрических пространств ранга $k$ совпадает с множеством решений этой системы неравенств (в дальнейшем будем ссылаться на нее, как на систему (1)).

Каждое неравенство системы (1) является строгим неравенством по крайней мере для одного решения этой системы: всегда присутствует решение $r(a, b)=1$ для всех 
$a \neq b$. О таких системах линейных однородных неравенств вида

$$
\begin{array}{r}
a_{1}^{1} x_{1}+a_{1}^{2} x_{2}+\cdots+a_{1}^{n} x_{n} \geqslant 0, \\
a_{2}^{1} x_{1}+a_{2}^{2} x_{2}+\cdots+a_{2}^{n} x_{n} \geqslant 0, \\
\cdots \cdots+\cdots+\cdots+\cdots+\cdots+a_{m}^{n} x_{n} \geqslant 0 \\
a_{m}^{1} x_{1}+a_{m}^{2} x_{2}+\cdots+
\end{array}
$$

известно следующее [6].

Если решения системы (2) рассматривать как векторы в $n$-мерном вещественном пространстве, то множество решений образует область размерности $n$, называемую конусом решений. Общее решение представимо в виде $\bar{x}=\alpha_{1} \bar{x}_{1}+\alpha_{2} \bar{x}_{2}+\cdots+\alpha_{N} \bar{x}_{N}$, где $\alpha_{1}, \ldots, \alpha_{N}$ - неотрицательные коэффициенты, $\bar{x}_{1}, \ldots, \bar{x}_{N}-$ минимальное множество векторов, назьваемое фундаментальной системой решений. Фундаментальная система единственна с точностью до коэффициентов, т.е. векторы $\bar{x}_{1}, \ldots, \bar{x}_{N}$ можно заменять только на коллинеарные. Векторы $\bar{x}_{1}, \ldots, \bar{x}_{N}$ (притом только они и коллинеарные им) не представимы в виде суммы неколлинеарных векторов из конуса решений. Лучи, определяемые векторами $\bar{x}_{1}, \ldots, \bar{x}_{N}$, и только они, являются ребрами конуса решений, т.е. получаются обращением в равенства $n-1$ линейно независимых неравенств системы.

Все ребра конуса можно найти с помощью следующей процедуры. Надо заменить все неравенства системы (2) на равенства:

$$
\begin{array}{r}
a_{1}^{1} x_{1}+a_{1}^{2} x_{2}+\cdots+a_{1}^{n} x_{n}=0, \\
a_{2}^{1} x_{1}+a_{2}^{2} x_{2}+\cdots+a_{2}^{n} x_{n}=0, \\
\cdots \cdots \cdots+\cdots \cdots \cdots+\cdots \cdots+\cdots+a_{m}^{n} x_{n}=0, \\
a_{m}^{1} x_{1}+a_{m}^{2} x_{2}+\cdots+
\end{array}
$$

и перебрать все подсистемы системы (3), ранг которых равен $n-1$. Каждая такая подсистема имеет фундаментальную систему из одного решения. Если это решение $\bar{x}$ удовлетворяет системе (2), то оно определяет ребро конуса; если оно удовлетворяет системе $(2)$ с противоположньми знаками неравенств ( " $\leqslant$ "), то $-\bar{x}$ определяет ребро конуса. В остальных случаях $\bar{x}$ не представляет интереса [6].

Для системы (1) отсюда следует, что для любого ранга $k$ существует конечньй и минимальньй набор пространств, порождающий все пространства данного ранга. Такой набор назовем базисом, а входящие в него пространства - базисными. Эти пространства и подобные им неразложимы. Любое другое пространство ранга $k$ разложимо и представимо как линейная комбинация базисных пространств, т.е. разлагается по базису с положительными коэффищиентами, хотя и необязательно единственным образом.

Очевидно, можно объявить базисным любое пространство из семейства подобных друг другу неразложимых пространств. Для определенности будем считать базисным такое пространство, в котором наименьшая ненулевая сторона равна 1.

Поскольку в системе (1) все коэффициенты целые (0 и \pm 1$)$, длины сторон в неразложимом пространстве должны находиться в целочисленных отношениях.

5. Базисы. Найдены базисы всех конечных псевдометрических пространств ранга $k \leqslant 7$. Не вдаваясь в процедуру поиска, изложим результаты.

Ранг 1. Представлен одноточечньм пространством, которое, очевидно, является базисом для самого себя. 


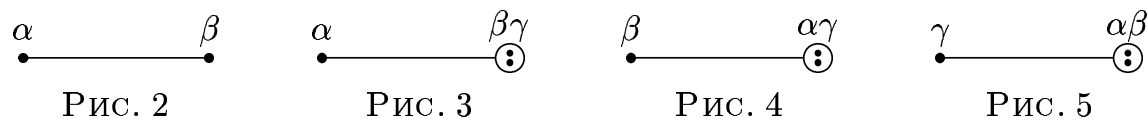

Ранг 2. Будем для определенности считать базисным пространством пару точек с расстоянием между ними 1 (рис. 2).

Ранг 3. Базис состоит из пространств, изображенных на рис. 3-5.

Дадим геометрическое доказательство тому, что любое метрическое пространство ранга 3 можно представить как линейную комбинацию этих пространств. Очевидно, любой метрической тройке точек $\alpha, \beta, \gamma$ с расстояниями между ними $r(\beta, \gamma)=x$, $r(\alpha, \gamma)=y, r(\alpha, \beta)=z$ можно поставить в соответствие плоский треугольник. Впишем в треугольник окружность, а расстояния от вершин до точек касания обозначим $a, b, c$ (рис. 6 ). Тогда $x=b+c, y=a+c, z=a+b$. Если величины $a, b, c$ взять в качестве коэффициентов в линейной комбинации пространств на рис. $3-5$ соответственно, то в результате получится исходная метрическая тройка, что и требовалось доказать.

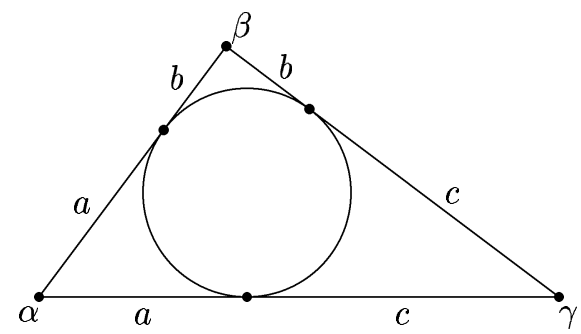

Рис. 6

Результат справедлив и для случая, когда треугольник вырождается в прямую или точку, т.е. любую псевдометрическую тройку можно разложить по базису с неотрицательными коэффициентами.

Справедливо и обратное: любой тройке неотрицательных чисел $a, b, c$ соответствует единственная псевдометрическая тройка со сторонами $a+b, b+c, a+c$. Неравенство треугольника, очевидно, всегда вьполняется.

Нетрудно проверить, что ни одно из пространств на рис. 3-5 не представимо в виде линейной комбинации двух остальньх, иными словами, эти пространства действительно образуют полную и минимальную порождающую систему, т.е. базис, ранга 3.

Ранг 4. Базис состоит из пространств, изображенных на рис. 7-13. Пространства на рис. 7-10 изоморфны, т.е. отличаются только расположением точек, совпадая по конфигурации. То же относится к пространствам на рис. 11-13. Можно сказать, что в ранге 4 существуют два базисных пространства с точностью до изоморфизма.

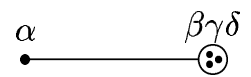

Рис. 7

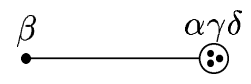

Рис. 8

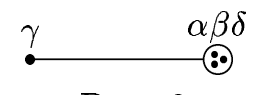

Рис. 9

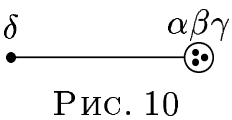

Рис. 10
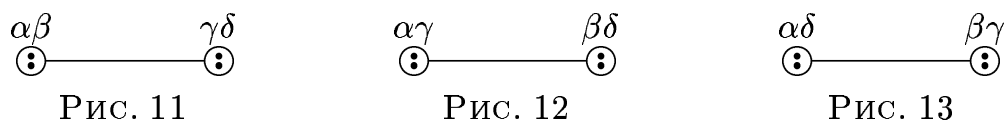
Пространства, состоящие из двух вершин с расстоянием между ними 1 , как на рис. $2-5$ и рис. $7-13$, будем назьвать разбиениями. Заметим, что в рангах $2,3,4$ базис является набором всевозможных разбиений. Далее будет доказано, что всякое разбиение неразложимо.

В ранге 4 разложение пространств по базису, вообще говоря, неоднозначно. Поскольку базисных пространств семь, а сторон в пространстве из четырех точек - шесть, то, определяя коэффициенты разложения, мы решаем систему из шести уравнений с семью неизвестными. Разложение будет неоднозначным и во всех последующих рангах.

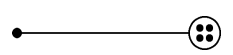

Рис. 14

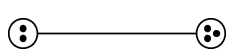

Рис. 15

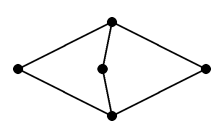

Рис. 16

Ранг 5. Базис с точностью до изоморфизма состоит из трех пространств (рис. 14-16). Здесь точки лишены обозначений, поскольку каждый граф изображает не отдельное пространство, а семейство изоморфных пространств.

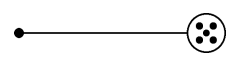

Рис. 17

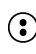

$$
\text { Рис. } 18
$$

(:)

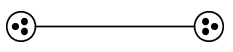

Рис. 19

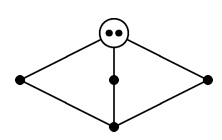

Рис. 20

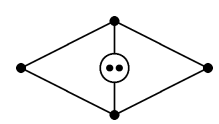

Р ис. 21

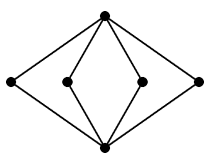

Рис. 22

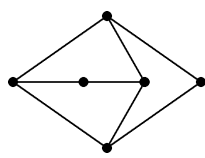

Рис. 23

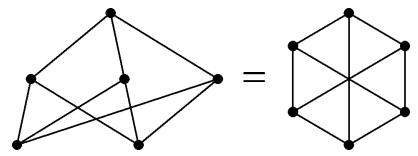

Рис. 24

Ранг 6. Базис с точностью до изоморфизма состоит из восьми пространств (рис. 17-24; знак равенства на рис. 24 означает, что оба способа изображения идентичны). Пространства на рис. 20, 21 отличаются от пространства на рис. 16 тем, что на месте точки располагается кратная вершина. Будем говорить, что пространства на рис. 20, 21 получены из пространства на рис. 16 удвоением точки. Далее будет доказано, что изменение кратности вершины (только не до 0: исчезновение вершины не считаем изменением ее кратности) не влияет на разложимость или неразложимость пространства.

Ранг 7. Здесь не изображены пространства с кратными вершинами, поскольку они получаются из базисных пространств ранга 6. Остаются с точностью до изоморфизма 29 пространств без кратных вершин (рис. 25-53). Здесь уже появляются пространства с ребрами разной длины (рис. 44-53). Длины ребер, отличные от 1 , отмечены соответствующими числами.

6. Признаки разложимости и неразложимости. Рангом 7 исчерпьвается перечень найденных базисов. В высших рангах полньй поиск не производился. Выведена только процедура определения разложимости любого конечного пространства и установлены отдельные признаки разложимости и неразложимости.

ТЕОРемА 1. При изменении кратности вериин разложимые псевдометрические пространства остаются разложимыми, а неразложимые-неразложимыми. 


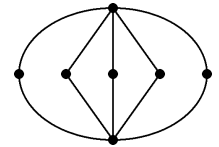

Рис. 25

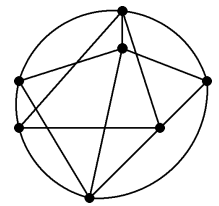

Рис. 30

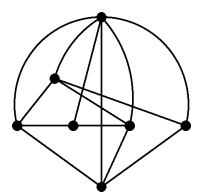

Рис. 35

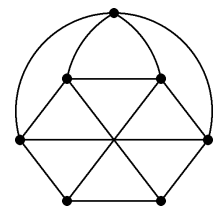

Рис. 39

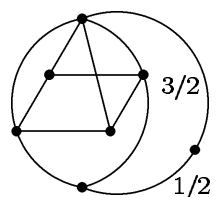

Рис. 44

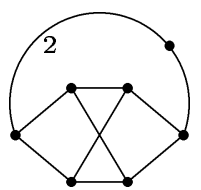

Рис. 49

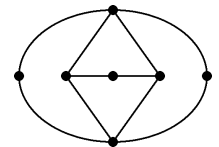

Рис. 26

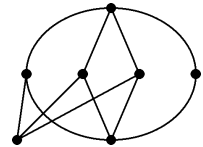

Рис. 27

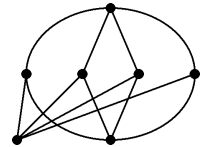

Рис. 28

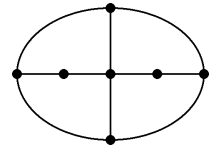

Рис. 29

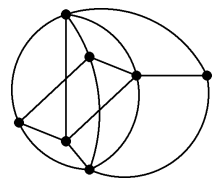

Рис. 31

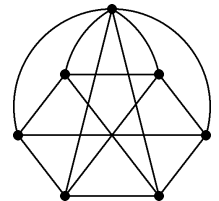

Рис. 32

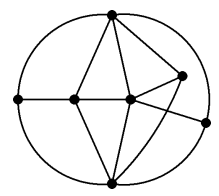

Рис. 33

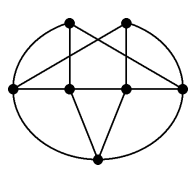

Рис. 37

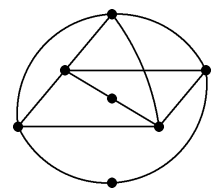

Рис. 42

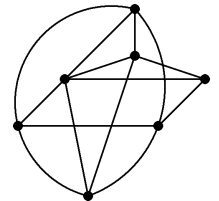

Рис. 34

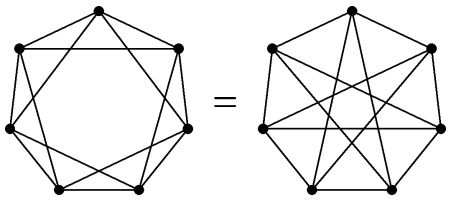

Рис. 36

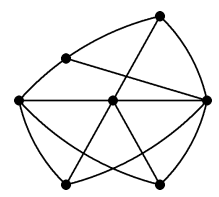

Рис. 40

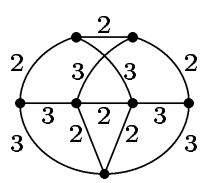

Рис. 45

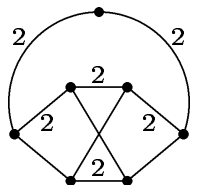

Рис. 50

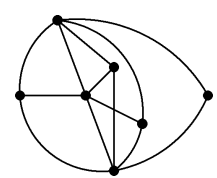

Рис. 41

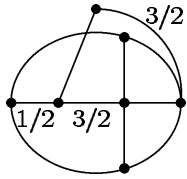

Рис. 46

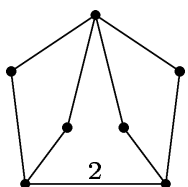

Рис. 51

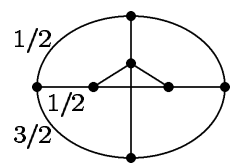

Рис. 47

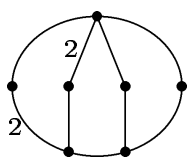

Рис. 52

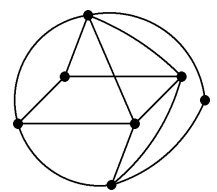

Рис. 38

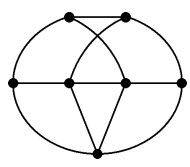

Рис. 43

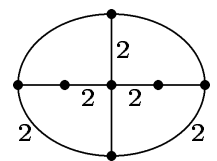

Рис. 48

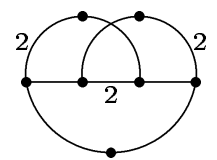

Рис. 53

ДокАЗАТЕльСтво. Докажем сначала, что неразложимость сохраняется при повышении кратности вершины на одну точку. На рис. 54 изображено неразложимое пространство $A a$, состоящее из вершины $a$ (неважно, сколько точек в нее входит) и остальной части $A$, изображенной обобщенно, без внутренних подробностей. Так же обобщенно изображен и комплект ребер, соединяющий вершину $a$ с частью $A$. Добавим к вершине $a$ точку $b$. Пусть получившееся пространство $A a b$ разлагается на $B a b$ и $C a b$. Очевидно, в обоих пространствах-слагаемых $r(a, b)=0$. Удалив из них по точке $b$, получим пространства $B a$ и $C a$. Их сумма составит $A a$. Так как $A a$ неразложимо, то $B a$ и $C a$ должны быть подобны. Тогда будут подобны $B a b$ и $C a b$, так как слившиеся вершины $a$ и $b$ дают одинаковые наборы расстояний. Иными словами, пространство $A a b$ неразложимо. 


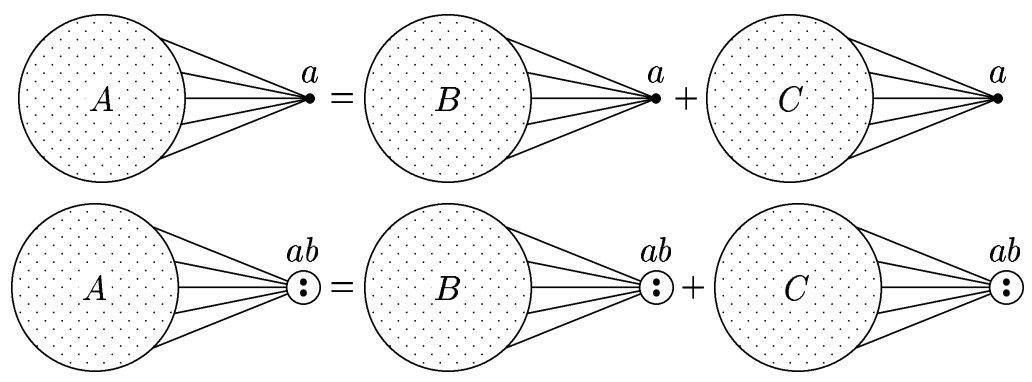

Рис. 54

Рассмотрим теперь неразложимое пространство $D a b$ с кратной вершиной $a b$. Удалим из нее точку $b$ и пространство $D a$ разложим на $E a$ и $F a$. Присоединим к этим пространствам по точке $b$, тогда они в сумме дадут $D a b$. Так как $D a b$ неразложимо, $E a b$ и $F a b$ подобны. Тогда подобны $E a$ и $F a$, значит $D a$ неразложимо.

Таким образом, неразложимость сохраняется при изменении кратности вершины на одну точку. Тогда неразложимость будет сохраняться и при изменении кратности произвольного числа вершин на произвольное число точек. При изменении кратности вершин должна сохраняться и разложимость: в противном случае мы могли бы получить разложимое пространство из неразложимого, изменив кратность вершин. Теорема доказана.

СлЕДСтвИЕ 1. Всякое разбиение неразложимо, так как любое разбиение отличается от неразложимого пространства на рис. 2 только кратностью вершин.

СледСтвиЕ 2. Все базисные пространства ранга $k$ с кратными вершинами можнно получить из базисных пространств ранга $k-1$ удвоением точек.

Таким образом, проверяя разложимость псевдометрического пространства $P$, можно заменить все его кратные вершины на одиночные и далее проверять разложимость соответствующего метрического пространства $M$. Если пространство $M$ неразложимо, то оно должно получаться как решение подсистемы из $n-1$ линейно независимых уравнений системы вида (3). Все равенства, существующие в пространстве $M$, очевидно, либо входят в эту подсистему, либо вытекают из нее. И обратно: в этой подсистеме не существует иных равенств, кроме тех, что вьполняются в пространстве $M$. Поэтому можно, считая стороны пространства $M$ неизвестными, составить систему из всех равенств типа $r(a, b)=r(a, c)+r(b, c)$, вьполняющихся в пространстве $M$ (общего вида не приводим, но будем ссылаться на нее, как на систему (4)).

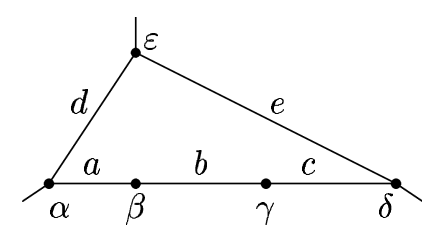

Рис. 55. $a+b+c=d+e$

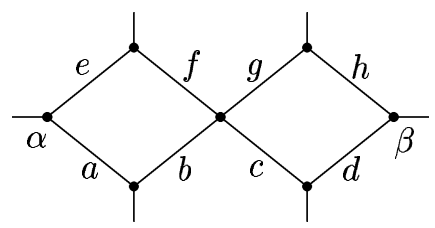

Рис. 56. $a+b+c+d=e+f+g+h$

Если в $M$ число вершин три и более, то систему (4) можно упростить, исключив составные стороны. Пусть, например, рис. 55 есть фрагмент графического изображения пространства $M$. Пусть $\alpha \delta$ - составная сторона. Она должна равняться кратчайшему маршруту, соединяющему точки $\alpha$ и $\delta$. Пусть таких маршрутов два: $\alpha \beta \gamma \delta$ и $\alpha \varepsilon \delta,-$ и 
состоят они из элементарных сторон $a, b, c, d, e$. В этом фрагменте вьполняются равенства

$$
\begin{gathered}
r(\alpha, \delta)=r(\alpha, \gamma)+c, \quad r(\alpha, \delta)=r(\beta, \delta)+a \\
r(\alpha, \delta)=d+e, \quad r(\alpha, \gamma)=a+b, \quad r(\beta, \delta)=b+c .
\end{gathered}
$$

Система (5) равносильна системе

$$
a+b+c=d+e, \quad r(\alpha, \delta)=a+b+c, \quad r(\alpha, \gamma)=a+b, \quad r(\beta, \delta)=b+c .
$$

Подобным образом можно преобразовать всю систему (4), выразив составные стороны через элементарные и приравняв друг к другу различные выражения для составных сторон. В системе останутся уравнения двух видов:

а) выражающие составные стороны через элементарные - по одному для каждой составной стороны;

б) приравниваюшие друг к другу различные кратчайшие маршруты между двумя вершинами.

Далее, из уравнений вида б) можно исключить те, в которых маршруты имеют общие средние точки. Пример показан на рис. 56 , где точки $\alpha$ и $\beta$ связаны кратчайшими маршрутами $a b c d$ и $e f g h$. Поскольку оба маршрута кратчайшие, $a+b=e+f$ и $c+d=g+h$. Так как эти уравнения обязательно должны присутствовать в преобразованной системе (4), вытекающее из них уравнение $a+b+c+d=e+f+g+h$ можно исключить. Иными словами, достаточно приравнять друг к другу лишь те маршруты, которыепри соединении образуют простой цикл (в котором не повторяются вершины и ребра) [5].

Можно, наконец, исключить уравнения вида а). Для нахождения элементарных сторон они не нужны, а когда элементарные стороны будут найдены, составные стороны можно будет вычислить непосредственно по графическому изображению.

Остается решить систему. Если получится множество решений с одной степенью свободы, то пространство $M$ неразложимо. В противном случае $M$ разложимо.

Результат сформулируем в виде теоремы.

ТЕОРема 2. Чтобы проверить разложимость конечного псевдометрического пространства с числом вериин не менее трех, мохсно, обозначив әлементарные стороны как неизвестные, составить все возмоэнные уравнения, в которых приравниваются друг $к$ другу образующие простой иикл кратчайшие марируты межсду двумя вершинами. Если система уравнений имеет множсество решений с одной степенью свободы, то пространство неразложсим. В противном случае оно разложимо.

Такую систему уравнений будем называть проверочной.

СЛЕДСТВИЕ. Если в псевдометрическом пространстве отсутствуют составные стороны, то оно разложимо.

Чтобы проверочная система имела с точностью до коэффищиента одно решение, необходимо, очевидно, чтобы каждая элементарная сторона входила хотя бы в одно уравнение. Для этого необходимо, чтобы в графическом изображении каждое ребро входило хотя бы в один простой цикл, иными словами, каждое ребро должно быть циклическим. Кроме того, в графическом изображении не должно быть разделяющих вершин. Разделяющая вершина - такая, при удалении которой граф распадается на несвязанные части [5]. Если в графе есть разделяюшая вершина, то он состоит по меньшей мере из двух 
частей, не связанных ни одним простым циклом. Тогда система уравнений распадется на независимые части, не содержащие общих переменных, и число степеней свободы в решении будет не меньше двух. Связный граф без разделяюших вершин назьвается блоком. Граф, состоящий из одного ребра, тоже считается блоком [5]. Доказано, что в блоке с числом вершин более двух все ребра циклические [5]. Сказанное обобщим в теореме.

Теорема 3. Графическое изображсение конечного неразлохсимого псевдометрического пространства долэсно быть блоком.

ПРимеР 1. Проверим разложимость пространства, изображенного на рис. 57. Составные стороны здесь $\alpha \beta, \gamma \delta, \delta \varepsilon, \gamma \varepsilon$. Им соответствуют уравнения проверочной системы

$$
\begin{gathered}
\alpha \beta: a+b=c+d=e+f, \quad \gamma \delta: a+c=b+d, \\
\delta \varepsilon: c+e=d+f, \quad \gamma \varepsilon: a+e=b+f .
\end{gathered}
$$

Решив систему, получим $a=b=c=d=e=f$. Система решается однозначно с точностью до коэффициента. Следовательно, проверяемое пространство неразложимо.

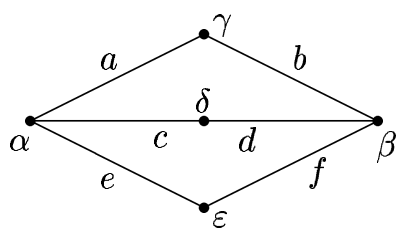

Рис. 57. $a=b=c=d=e=f=1$

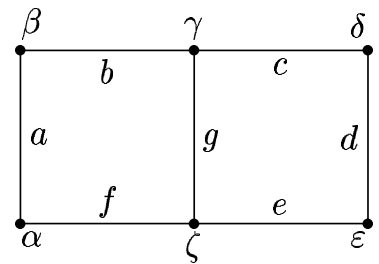

Рис. 58. $a=b=c=d=e=f=g=1$

ПРимеР 2. Проверим разложимость пространства, изображенного на рис. 58. Составные стороны здесь $\alpha \gamma, \beta \zeta, \gamma \varepsilon, \delta \zeta, \alpha \delta, \beta \varepsilon, \alpha \varepsilon, \beta \delta$. Сторона $\alpha \varepsilon$ не дает уравнений, так как точки $\alpha$ и $\varepsilon$ соединены только одной кратчайшей цепью $\alpha \zeta \varepsilon$; то же относится к стороне $\beta \delta$. Точки $\alpha$ и $\delta$ соединены тремя кратчайшими цепями: $a b c, f g c, f e d$, но простой цикл образует лишь пара $a b c, f e d$. Она и дает уравнение. То же относится к стороне $\beta \varepsilon$. Получаем проверочную систему

$$
\begin{array}{cl}
\alpha \gamma: a+b=f+g, \quad \beta \zeta: a+f=b+g, & \gamma \varepsilon: c+d=g+e, \\
\delta \zeta: c+g=d+e, \quad \alpha \delta: a+b+c=d+e+f, & \beta \varepsilon: b+c+d=a+f+e .
\end{array}
$$

Решив систему, получим $a=d=g, b=f, c=e$. Решение имеет три степени свободы. Следовательно, проверяемое пространство разложимо.

\section{СПИСОК ЦИТИРОВАННОЙ ЛИТЕРАТУРЫ}

[1] Келли Дж. Л. Общая топология. М.: Наука, 1981.

[2] Куратовский К. Топология. Т. 1. М.: Мир, 1966.

[3] Kaplansky I. Set Theory and Metric Spaces. New York: Chelsea, 1977.

[4] Зыков А. А. Основы теории графов. М.: Наука, 1987.

[5] Оре О. Теория графов. М.: Наука, 1980.

[6] Беклемишев Д. В. Дополнительные главы линейной алгебры. М.: Наука, 1983.

Институт генетики АН Республики Молдова, г. Кишинев

Поступило 01.12 .95

Исправленный вариант 05.09.97 